\title{
La evaluación de los trastornos de la personalidad según el DSM-5: Recursos y limitaciones
}

\author{
Assessment of personality disorders according to DSM-5: Available instruments and \\ limitations
}

\author{
Enrique Esbec \\ Universidad Complutense de Madrid, España \\ Enrique Echeburúa \\ Facultad de Psicología, Universidad del País Vasco, España
}

(Rec: 16 junio 2014 / Acept: 17 octubre 2014)

\begin{abstract}
Resumen
El objetivo de este artículo es hacer una revisión sobre los instrumentos de medida disponibles en español para la evaluación de los trastornos de personalidad (TP) según el DSM-5. El enfoque actual de los TP figura en la Sección II del DSM-5 y no registra cambios respecto al DSM-IV. Sin embargo, se presenta en la Sección III un modelo alternativo dimensional-categorial. Las categorías diagnósticas derivadas de este modelo en la Sección III incluyen sólo los TP antisocial, evitativo, límite, narcisista, obsesivo-compulsivo y esquizotípico y se evalúan con entrevistas clínicas estructuradas, tales como el SCID-II o el IPDE. A su vez, los rasgos de personalidad patológicos se evalúan con autoinformes diseñados específicamente para el sistema clasificatorio del DSM-5, como el PID-5, o con autoinformes más genéricos, como el MMPI-2-RF, el MCMI-III o el PAI. Sin embargo, los autoinformes presentan una tendencia al sobrediagnóstico. Se desarrollan algunas sugerencias para la investigación futura.

Palabras clave: trastornos de personalidad, DSM-5, evaluación, entrevistas estructuradas y autoinformes.
\end{abstract}

\begin{abstract}
This paper aims at reviewing interviews and self-reports avalaible in Spanish for the assessment of personality disorders (PD) according to DSM-5. The current approach to PD appears in Section II of DSM-5 and has not changed from that in DSM-IV, except that they are moved to the main disorder section from Axis II. However, there is an alternative model developed in Section III. PD are characterized by impairments in personality functioning and by pathological personality traits. Diagnostic categories derived from this model include only antisocial, avoidant, borderline, narcissistic, obsessive-compulsive and schizotypal personality disorders. Diagnostic categories are assessed with structured clinical interviews, such as SCID-II or IPDE. In turn pathological personality traits are assessed with self-reports specifically designed for DSM-5 alternative dimensional model, such as PID-5, or with more general self-reports, such as MMPI-2-RF, MCMI-III or PAI. Self-reports have a tendency to over-diagnose personality disorders. Suggestions for future research are outlined.

Keywords: Personality disorders, DSM-5, assessment, structured interviews, self-reports.
\end{abstract}

\footnotetext{
Correspondencia:

Enrique Echeburúa. Facultad de Psicología, Universidad del País Vasco. Avda. de Tolosa, 70. 20018 San Sebastián (España).E-mail: enrique.echeburua@ehu.es
} 


\section{Introducción}

La evaluación de la personalidad y su gama de anormalidad es uno de los elementos críticos en un examen psiquiátrico/ psicológico. Sin embargo, se omite con frecuencia en las evaluaciones clínicas, que rara vez la evalúan formalmente. A nivel clínico, el desinterés mostrado por los profesionales hacia los trastornos de personalidad (TP) deriva de que éstos tienden a ser permanentes y se muestran resistentes al tratamiento. Muy a menudo, el diagnóstico de los TP solo se toma en consideración cuando la evolución del trastorno mental en tratamiento es insatisfactoria (Esbec y Echeburúa, 2011).

El DSM-5 (American Psychiatric Association, 2013) incluye dos tipos de definición y de sistemas de valoración para los TP. Por un lado, en la Sección II (clasificación oficial) se presenta una definición categorial de los TP, prácticamente idéntica a la anterior del DMS-IV, que abarca los diez trastornos específicos tradicionales, más algunos especificados y otro no especificado. Por otro lado, en la Sección III (dedicada a los trastornos que requieren más estudio) se propone una definición basada en rasgos de personalidad y en el funcionamiento de la misma, de valoración dimensional, que incluye seis tipos específicos de personalidad (esquizotípico, límite, antisocial, narcisista, obsesivo-compulsivo y ansioso-evitativo), cuya tipificación requiere la evaluación de 25 rasgos de personalidad incluidos en 5 dimensiones de orden superior (Echeburúa, Salaberría y Cruz-Sáez, 2014).

Conocer el nivel de funcionamiento de la personalidad de un individuo y su perfil de rasgos patológicos proporciona al clínico una rica base de información para la planificación del tratamiento y para la predicción del curso de muchos trastornos mentales, además del TP en sí mismo. Por lo tanto, la evaluación del funcionamiento de la personalidad y de los rasgos de personalidad patológicos puede ser relevante tanto si una persona tiene un TP como si no lo tiene.

La evaluación diagnóstica se enfrenta a la divergencia de opiniones entre los que son partidarios de las entrevistas abiertas y los que creen más en la administración de los exámenes estandarizados. Aunque algunos profesionales basan su diagnóstico en la información proporcionada por sus pacientes y en el examen directo de cómo los pacientes se muestran emocional y conductualmente, otros prefieren usar las pruebas estandarizadas con preguntas directivas.

No hay todavía ningún instrumento completamente fiable para el diagnóstico de los TP. De hecho, el índice de fiabilidad diagnóstica (concordancia) de las entrevistas estructuradas es moderado, con un índice kappa de algo más de 0.5. A su vez, los autoinformes han demostrado ser poco eficaces en la identificación de estos trastornos.

La desaparición del Eje II en el DSM-5, lo que implica la consideración de los TP como trastornos mentales de pleno derecho, obliga a una evaluación detallada de los mismos. El objetivo de este artículo es hacer una presentación de las entrevistas y autoinformes disponibles para la población de habla española, así como elaborar un análisis crítico y presentar las líneas de investigación futuras.

\section{Valoración de los TP en la Sección II del DSM-5}

La Sección II del DSM-5 propone una valoración de los TP basada más en conductas que en rasgos o disposiciones. Por lo tanto, en este formato es esencial conocer en profundidad la psicobiografía y la historia clínica, así como los antecedentes del paciente. Habitualmente, el clínico debe buscar información adicional en informadores externos y en documentos. No obstante, como pruebas complementarias cuentan con buena fiabilidad y validez las entrevistas estructuradas, tales como el SCID-II (Spitzer, Williams y Gibbon, 1989) y el IPDE (Loranger, 1995). A su vez, los tests de personalidad pueden ser de alguna utilidad, pero, por sí solos, no sirven para efectuar el diagnóstico clínico.

Las entrevistas estructuradas se han desarrollado para aumentar la fiabilidad diagnóstica a través de la estandarización del proceso de evaluación y para mejorar la validez diagnóstica mediante la aplicación de los criterios del DSM.

\section{International Personality Disorder Examination (IPDE)}

El Examen Internacional para los TP (IPDE) (Loranger, 1995; versión española de López-Ibor, Pérez Urdániz y Rubio, 1996) es un instrumento diagnóstico basado en una entrevista clínica semiestructurada, que además es compatible con los criterios de valoración de la CIE-10 y del DSM-IV. La entrevista es la más utilizada de su clase y es la única entrevista para TP sobre la base de pruebas de campo en todo el mundo.

El manual del IPDE contiene tanto un cuestionario de detección como una entrevista semiestructurada. El cuestionario de detección es una forma auto-administrada que contiene 77 ítems en la versión para el DSM y 59 para la versión de la CIE-10. El paciente responde Verdadero o Falso para cada elemento y puede completar el cuestionario en menos de 15 minutos. De este modo, el clínico puede identificar aquellos pacientes cuyos resultados sugieren la 
Tabla 1. Criterios generales y categorías diagnósticas de los TP según la valoración alternativa del DSM-5 (Sección III).

\begin{tabular}{ll}
\hline Criterios & Categorías diagnósticas \\
\hline $\begin{array}{l}\text { A. Deterioro moderado o grave en el funcionamiento (propio/interpersonal) de la } \\
\text { personalidad. }\end{array}$ & Trastorno antisocial de la personalidad. \\
$\begin{array}{l}\text { B. Uno o más rasgos de personalidad patológicos. } \\
\text { C. Rasgos y deterioro en el funcionamiento relativamente permanentes e inflexibles } \\
\text { en diferentes situaciones sociales y personales. }\end{array}$ & Trastorno de la personalidad por evitación. \\
$\begin{array}{l}\text { D. Relativamente estables en el tiempo. Comienzo en la adolescencia o en el inicio personalidad. } \\
\text { de la edad adulta. }\end{array}$ & Trastorno narcisista de la personalidad. \\
$\begin{array}{l}\text { E. No explicados mejor por otro trastorno mental. } \\
\begin{array}{l}\text { F. No atribuibles a sustancias adictivas o a enfermedades médicas. } \\
\text { G. No comprendidos mejor por la edad (desarrollo) o el entorno sociocultural. }\end{array}\end{array}$ & Trastorno de la personalidad obsesivo-compulsivo. \\
\hline
\end{tabular}

presencia de un trastorno de personalidad (más de tres criterios para cada TP). Si el cuestionario genera la sospecha de uno o varios TP, se debe pasar la entrevista completa, lo que permitirá al clínico evaluar la presencia o no de algún TP (diagnóstico definitivo o probable).

Las preguntas de la entrevista están dispuestas en un formato equilibrado entre una entrevista clínica espontánea y los requisitos de la normalización y la objetividad. Las preguntas están organizadas en secciones (por ejemplo, información relacionada con el trabajo, uno mismo, control de impulsos y relaciones interpersonales) que incluyen preguntas abiertas al principio de cada sección. Para cada pregunta de la entrevista IPDE, el TP correspondiente y el criterio diagnóstico específico se identifican con las directrices precisas de puntuación. Además, al menos uno de los criterios debe haber estado presente antes de los 25 años. El clínico registra las puntuaciones en la hoja de respuestas y elabora el perfil de puntuación dimensional.

El IPDE es una herramienta fácil de usar y clínicamente significativa. Se ha demostrado la fiabilidad inter-evaluadores y la estabilidad temporal, que es más o menos similar a la de los instrumentos utilizados para el diagnóstico de psicosis, alteraciones del estado de ánimo, trastornos de ansiedad y abuso de sustancias. EL IPDE completo puede descargarse desde la web de la OMS.

\section{Structured Clinical Interview for Personality Disorders (SCID-II)}

La Entrevista Clínica Estructurada para los Trastornos de Personalidad del DSM-IV (SCID-II) (Spitzer et al., 1989) es una entrevista semiestructurada que puede usarse para formular diagnósticos de forma tanto categorial (presente o ausente) como dimensional (reseñando el número de criterios para cada TP que han sido codificados como " 3 ").

Uno de los objetivos en la elaboración de la SCID fue el promover un instrumento eficiente y de fácil manejo, de tal forma que las ventajas de la entrevista estructurada pudieran ser aplicadas en el ámbito clínico. La SCID puede ser utilizada en los centros de salud mental, adonde acuden casos complejos que requieren un diagnóstico fiable y preciso.

\section{Valoración de los TP en la Sección III del DSM-5}

En la Sección III del DSM-5 se propone un modelo híbrido categórico-dimensional para el diagnóstico de los TP. Este modelo incluye un conjunto de rasgos y dominios de la personalidad que, junto con el mayor o menor deterioro en el funcionamiento de la personalidad, se configuran para categorizar uno de los seis TP propuestos (tabla 1). Este enfoque es más flexible, más específico y presta una mayor atención al nivel de gravedad (Skodol et al., 2011).

El evaluador debe prestar atención al grado de deterioro en el funcionamiento de la personalidad en el ámbito intrapersonal e interpersonal, a los rasgos y dominios anómalos de la personalidad (así como a su severidad) y a la posible presencia de alguno de los TP definidos.

A pesar de la reticencia de muchos profesionales a utilizar el diagnóstico antes de los 18 años, los TP se pueden diagnosticar ya en la adolescencia. De hecho, la patología de la personalidad parece ser más alta antes de los 20 años, con un descenso de la mayoría de las características patológicas con el transcurso del tiempo. Este diagnóstico temprano puede ser de ayuda para la adopción adecuada de decisiones terapéuticas.

Las medidas de evaluación desempeñan un papel muy importante para el diagnóstico inicial y para la toma de decisiones clínicas a lo largo del tratamiento. El clínico debe valorar el nivel de funcionamiento de la personalidad de acuerdo con la escala que se adjunta en el DSM-5. Para ello debe basarse en la anamnesis, exploración e información complementaria, ya que no se ha desarrollado todavía ningún instrumento objetivo. Si existe afectación moderada o severa del funcionamiento, se procederá a pasar el PID-5 (véase más adelante) en la versión reducida, debiendo ser 
cumplimentado por el paciente y por un informador (por ejemplo, la pareja). En el caso de existir una afectación de moderada a severa en algún dominio, hay que pasar la prueba completa (paciente e informador) para obtener las 25 facetas-rasgo. La evaluación de los 5 dominios es una opción clínica aceptable cuando sólo se necesita un retrato general de la personalidad del paciente. Sin embargo, si los problemas basados en la personalidad son el centro de la evaluación o tratamiento, entonces será importante estimar todas las facetas-rasgo de los individuos, así como los dominios.

Debido a que los rasgos de personalidad se distribuyen de forma continua en la población, se pueden comparar los rasgos de personalidad de un sujeto concreto con los de la población a que pertenece.

\section{Valoración del funcionamiento de la personalidad}

Debido a que puede haber un mayor o menor grado de perturbación, cada paciente debe ser evaluado en el continuo compuesto por los siguientes niveles de funcionamiento: personal (identidad y autodirección) e interpersonal (empatía e intimidad). El criterio del funcionamiento es un criterio "llave", es decir, debe haber una afectación moderada o grave en al menos dos de ellos para poder efectuar el diagnóstico, con arreglo al grado de deterioro de la escala de valoración del DSM-5, que establece 5 niveles, desde "no menoscabo" (nivel 0) a "deterioro extremo" (nivel 4).

La identidad está relacionada con la mismidad, la continuidad en el tiempo del individuo y el reconocimiento de los otros de estas cualidades. Así, por ejemplo, un adolescente con problemas de identidad se describe de manera superficial y poco elaborada ("yo soy tímido, yo necesito a mi novia..."), a pesar de contar con una inteligencia normal, y puede mostrar síntomas depresivos severos o síntomas disociativos ( "no puedo sentir mi cuerpo...", "me veo a mí mismo desde fuera, como en una película..."), así como tener una imagen muy inestable e incoherente de sí mismo ("no tengo ni idea de quién soy...”). En estos casos, la relación interpersonal sirve sólo para estabilizar sus sentimientos de soledad profunda y es intercambiable (no importa si es la madre o la pareja quien está presente; lo más importante es que una persona se encuentre disponible) (Schmeck et al., 2013). La autodirección puede evaluarse con el Inventario de Temperamento y Carácter Revisado (TCI- R) (Cloninger, 2000), adaptado a la población española por Gutiérrez-Zotes et al. (2004).

A su vez, la empatía puede medirse a través del Índice de Reactividad Interpersonal (IRI) (Davis, 1983; versión española de Mestre, Frías y Samper, 2004), que permite evaluar la disposición empática a través de cuatro factores, dos cognitivos y dos emocionales: a) Toma de perspectiva (PT) (habilidad para comprender el punto de vista de la otra persona); b) Fantasía (FS) (tendencia a identificarse con personajes del cine y de la literatura); c) Preocupación empática (EC) (sentimientos de compasión, preocupación y cariño por otros); y d) Malestar personal (PD) (sentimientos de ansiedad y malestar que el sujeto manifiesta al observar las experiencias negativas de los demás). Otro instrumento de interés es el Test de Empatía Cognitiva y Afectiva (TECA) (López-Pérez, Fernández-Pinto y Abad, 2008), que es una medida global de la empatía compuesta por 33 ítems y que abarca cuatro factores: a) Adopción de perspectivas (capacidad intelectual o imaginativa de ponerse en el lugar de otra persona); b) Comprensión emocional (capacidad de reconocer y comprender los estados emocionales, las intenciones y las impresiones de las otras personas); c) Estrés empático (capacidad de compartir las emociones negativas de otra persona); y d) Alegría empática (capacidad de compartir las emociones positivas de otra persona) (Loinaz, Echeburúa y Ullate, 2012).

Como la mayoría de las tendencias humanas, el funcionamiento de la personalidad se distribuye en un continuo. El funcionamiento y la adaptación son formas características de los individuos de pensar y entenderse a sí mismos y sus interacciones con los demás. Así, una persona con funcionamiento óptimo tiene su mundo psicológico bien integrado, lo cual incluye un concepto de sí mismo básicamente positivo, una vida regulada emocionalmente y la capacidad de comportarse con relaciones interpersonales recíprocas y satisfactorias. Por el contrario, un individuo con una patología grave de la personalidad tiene un mundo psicológico empobrecido, desorganizado y/o en conflicto, que incluye un concepto de sí mismo mal adaptado, una propensión a las emociones negativas y una deficiente capacidad para las relaciones interpersonales.

Según la Escala de Valoración del Funcionamiento de la Personalidad (LPFS) del DSM-5, una personalidad adaptativa tiene la conciencia permanente de un yo único, manteniendo roles apropiados (Identidad), aspirando a metas razonables basadas en una evaluación realista de las capacidades personales (Autodirección), comprendiendo con precisión las experiencias y motivaciones de los demás en la mayoría de las situaciones (Empatía) y manteniendo relaciones satisfactorias y duraderas tanto en la vida personal como en la comunitaria (Intimidad). En esta escala, el clínico selecciona el nivel actual de deterioro en el funcionamiento de la personalidad, que es utilidad para el diagnóstico de 
un TP en un momento dado El LPFS también puede ser utilizado como un indicador global del funcionamiento de la personalidad sin especificación de un diagnóstico de TP o en el caso de que el deterioro de la personalidad sea subumbral para un diagnóstico de trastorno.

\section{Valoración de los rasgos de la personalidad}

La sección III del DSM-5 incluye la valoración de 5 dimensiones o dominios generales de personalidad y de 25 dimensiones más específicas (facetas-rasgo), que son variantes desadaptativas de los cinco dominios ampliamente validados en el modelo de los "cinco grandes" o de "los cinco factores" (tabla 2).

Los rasgos de personalidad incluidos abarcan un espectro con dos polos opuestos: afectividad negativa versus estabilidad emocional; desapego versus extraversión; antagonismo versus amabilidad; desinhibición versus escrupulosidad; y psicoticismo versus lucidez. Aunque el DSM-5 se centra en los rasgos de personalidad relacionados con la psicopatología (no saludables), pueden valorarse los rasgos de personalidad resiliente identificados como los polos opuestos de estos rasgos (es decir, estabilidad emocional, extraversión, amabilidad, responsabilidad y lucidez). Su presencia puede reducir en gran medida los efectos de los trastornos mentales, así como facilitar el afrontamiento y la recuperación de lesiones traumáticas y otras enfermedades médicas.

Sin ánimo de ser exhaustivos, se señalan a continuación los instrumentos de evaluación más útiles, con buenas propiedades psicométricas y con versiones en español.

a. PID-5 (The Personality Inventory for DSM-5)

El PID-5 (Krueger, Derringer, Markon, Watson y Skodol, 2010) es la medida del modelo dimensional de 25 rasgos propuesta para el DSM-5. Existen varias formas disponibles on-line en el dominio DSM5.org de la APA: a) Formato de 220 elementos para adultos (25 facetas-rasgo y 5 dominios); b) Formato breve de 25 ítems centrado en los 5 dominios; y c) Formato para niños de 11-17 años. En la página web viene explicado el sistema de corrección, así como la forma de prorratear los ítems ausentes.

La versión completa para adultos consta de 220 ítems valorados en una escala Likert como 0 (muy a menudo o falso), 1 (algunas veces o un tanto falso), 2 (a veces o algo cierto) y 3 (muy verdadero o frecuentemente cierto). Este instrumento contiene escalas para las 25 facetas-rasgo incluidas dentro del modelo. Cada faceta es evaluada por 4 a 14 elementos.

Si hay más de un $25 \%$ de elementos dentro de una faceta característica que se quedan sin respuesta, la puntuación de la faceta correspondiente no debe ser puntuada. Por lo tanto, la persona debe ser animada a completar todos los elementos de la prueba. Sin embargo, si el 25\% o menos de los ítems quedan sin respuesta para una faceta específica, se puede prorratear la puntuación de la faceta. Las puntuaciones de dominio no deben ser estimadas si cualquiera de las tres puntuaciones de la faceta que contribuyen a ese dominio no se puede calcular debido a la ausencia de respuesta. $\mathrm{Si}$ el resultado final es una fracción, se debe redondear al número entero más próximo.

En el formato breve (25 ítems) se obtienen sólo los 5 dominios de la personalidad. Así, el dominio afectividad negativa se consigue con los siguientes ítems (que hay que valorar en un rango de 0 a 3): "Me preocupo casi por todo; $a$ menudo me emociono fácilmente sin razón aparente; tengo miedo de estar solo en la vida más que cualquier cosa; me quedo bloqueado cuando hago las cosas, incluso cuando

Tabla 2. Dominios de orden superior y facetas de personalidad en el DSM-5 (Sección III)

Dominios de orden superior Facetas-rasgo de la personalidad

Labilidad emocional, ansiedad, inseguridad de separación, sumisión, hostilidad, perse-

Afectividad negativa verancia, depresividad (común a otros dominios), suspicacia (común a otros dominios), afectividad restringida (común a otros dominios).

Desapego

Antagonismo

Desinhibición

Psicoticismo
Retraimiento social, evitación de la intimidad, anhedonia, depresividad, afectividad restringida, suspicacia.

Manipulación, falta de honradez, grandiosidad, búsqueda de atención, insensibilidad, hostilidad (común a otros dominios).

Irresponsabilidad, impulsividad, distraibilidad, temeridad, carencia de perfeccionismo. Creencias y experiencias extrañas, excentricidad, desregulación cognitiva y perceptiva.
Número de

rasgos

(total: 25)

\section{6}

6

5

3 
está claro que van a funcionar; me irrito con facilidad por cualquier cosa".

Diferentes estudios han mostrado convergencia entre los factores y rasgos del PID-5 con el MMPI-2 PSY-5 (Anderson et al., 2013; Sellbom, Anderson y Bagby, 2013), con el NEOPI-R (Gore y Widiger, 2013; Thomas et al., 2013; Bastiaansen et al., 2013), con el Inventario de Problemas Interpersonales (Wright et al., 2012), con el modelo HEXACO (Ashton et al., 2012) y con el PAI (Hopwood et al., 2013), entre otros.

b. MMPI-2-RF (Minnesota Multiphasic Personality Inventory-2-RF)

El MMPI-2-RF (Ben-Porath y Tellegen, 2008; adaptación española de Santamaría, 2009), con 338 ítems, consta de 50 escalas organizadas conceptual y jerárquicamente, vinculadas a modelos actuales de personalidad y psicopatología, con mejores propiedades psicométricas de fiabilidad y validez que las versiones anteriores. Consta de escalas de validez (8); dimensiones globales (3); escalas clínicas reestructuradas (rc) (9); escalas de problemas específicos (23); escalas somáticas/cognitivas (5); escalas de internalización (9); escalas de externalización (4); escalas interpersonales (5); escalas de intereses (2); y escalas de personalidad psicopatológica (5) (PSY-5).

Las puntuaciones del MMPI-2-RF, especialmente las 5 escalas de personalidad patológica, convergen con el sistema pentafactorial de evaluación de la personalidad incluido en el DSM-5, así como con los trastornos de la Sección III. Esta versión del MMPI incluye escalas más claramente identificadas y estructuradas, y supone una reducción en el número de ítems (338).

Por lo tanto, el MMPI-2-RF es un instrumento potencialmente útil en la evaluación de la psicopatología de la personalidad, tal como se conceptualiza en la Sección III del DSM-5. De hecho, el MMPI-2 es la medida de personalidad más empleada por los psicólogos clínicos y los neuropsicólogos, siendo además parte habitual del proceso de evaluación forense de adultos por las escalas de sinceridad incluidas.

\section{c. MCMI-III (Inventario Clínico Mutiaxial de Millon)}

El MCMI-III (Millon, Millon, Davis y Grossman, 2006; adaptación española de Cardenal y Sánchez, 2007) consta de 175 ítems de respuesta dicotómica (verdadero o falso), 4 índices que permiten evaluar la validez del protocolo (sinceridad, validez, deseabilidad social y devaluación) y 24 escalas organizadas en función de la severidad. Estas escalas incluyen 11 patrones clínicos de personalidad de severidad moderada (esquizoide, evitativo, depresivo, dependiente, histriónico, narcisista, antisocial, sádico, compulsivo, negativista y masoquista), 3 patrones de personalidad graves (esquizotípico, límite y paranoide), 7 síndromes clínicos de gravedad moderada (ansiedad, trastorno somatoforme, trastorno bipolar, distimia, abuso de alcohol, abuso de drogas y trastorno de estrés postraumático) y 3 síndomes clínicos severos (trastorno del pensamiento, depresión mayor y trastorno delirante).

El enfoque de Millon para la exploración e identificación de dificultades emocionales y personales se ha convertido en un importante referente en el campo de la psicopatología. El inventario, que es un intento ambicioso para evaluar los síndromes clínicos del Eje I y los TP del Eje II del DSM-IV, se apoya en un nuevo marco teórico: la teoría evolutiva. Con la finalidad de optimizar la correspondencia con el DSM-IV se han sustituido 95 elementos y se han añadido dos nuevas escalas: un patrón de personalidad clínico (depresivo) y un nuevo síndrome (estrés postraumático).

El inventario tiene buenas propiedades psicométricas, pero, sin embargo, las escalas por sí mismas carecen de validez suficiente para hacer un diagnóstico de los TP (Rogers, Salekin y Sewell 1999).

\section{d. PAI (Personality Assessment Inventory)}

El Inventario de Evaluación de la Personalidad (PAI) (Morey, 1991 y 2007; adaptación española de OrtizTallo, Santamaría, Cardenal y Sánchez, 2011), que tiene 344 ítems, permite una evaluación comprehensiva de la personalidad y la psicopatología en adultos mediante 22 escalas: 4 escalas de validez (inconsistencia, infrecuencia, impresión negativa e impresión positiva), 11 escalas clínicas (quejas somáticas, ansiedad, trastornos relacionados con la ansiedad, depresión, manía, paranoia, esquizofrenia, rasgos límites, rasgos antisociales, problemas con el alcohol y problemas con las drogas), 5 escalas de consideraciones para el tratamiento (agresión, ideaciones suicidas, estrés, falta de apoyo social y rechazo al tratamiento) y 2 escalas de relaciones interpersonales (dominancia y afabilidad). Además, incluye 30 subescalas que proporcionan una información más pormenorizada. En el ámbito forense el PAI es utilizado tanto para el screening y diagnóstico como para la detección de grupos forenses específicos (p. ej., valoración de peligrosidad, custodia de menores, psicopatía, maltrato, etcétera) o en el ámbito de la selección de personal (p. ej., para militares y policías).

El PAI destaca por su claridad en la interpretación y la exhaustiva información que proporciona, por aquellos ítems críticos que requieren la atención inmediata del profesional. La amplia cantidad de información clínicamente relevante que ofrece lo convierte en una excelente opción para la 
evaluación de la psicopatología de adultos en múltiples contextos.

El PAI ofrece algunas ventajas sobre el MMPI-2: a) hay cuatro opciones de respuesta a los ítems frente al MMPI-2, que sólo ofrece dos; b) los ítems son más fácilmente comprensibles; y c) las escalas del PAI son más directamente relevantes a los criterios DSM que las del MMPI (Esbec y Robles, 2013).

\section{Evaluación de las categorías diagnósticas}

Las 25 facetas-rasgo específicas que se agrupan en los 5 dominios de orden superior (afectividad negativa, desapego, antagonismo, desinhibición y psicoticismo) se utilizan para «componer» los TP categorialmente. El DSM-5 (sección III) propone seis categorías específicas de TP definidos dimensionalmente por sus rasgos correspondientes: antisocial, límite, narcisista, evitativo, obsesivo-compulsivo y esquizotípico.

Los otros TP específicos que contemplaba el DSMIV y que siguen en la sección II del DSM-5 (esquizoide, paranoide, histriónico y dependiente), los demás TP en estudio (dependiente, pasivo-agresivo), el trastorno psicopático/sádico y la categoría residual de TP no especificado desaparecen de esta versión y deberían evaluarse, como en el caso de los TP, con una descripción detallada de las anomalías en el funcionamiento personal e interpersonal y con una valoración dimensional de los rasgos y dominios de la personalidad.

En el DSM-5 se necesita una valoración politética de los rasgos desadaptativos. Así, para cumplimentar el criterio B se requiere una afectación moderada o severa de un número determinado de facetas de personalidad: en el trastorno antisocial, seis o más de un total de siete facetas; en el evitativo, tres o más de un total de cuatro; en el límite, cuatro o más de un global de siete (pero exigiendo que se dé alguno de los tres rasgos esenciales); en el narcisista, los dos rasgos propuestos; en el obsesivo-compulsivo, tres o más de los cuatro rasgos desadaptativos que lo conforman; y en el esquizotípico, cuatro o más de los seis rasgos propuestos.

\section{Evaluación de los TP en el contexto forense. Evaluación de la psicopatía [PCL-R (Psychopathy Check List-Revised)]}

En el DSM-5 se advierte que el uso del manual debe efectuarse con especial precaución en contextos judiciales o forenses, y especialmente por lo que se refiere a la utilización de las pruebas psicodiagnósticas.

La evaluación de la personalidad se utiliza en un amplio rango de contextos legales y forenses como parte del proceso de toma de decisiones legales, desde la responsabilidad criminal hasta la custodia infantil, la evaluación del riesgo de violencia o la valoración del daño psíquico (Melton, Petrila, Poythress y Slobogin, 2007). En el ámbito penitenciario, la evaluación de la personalidad puede suponer un recurso crítico para detectar a los individuos violentos, la conducta autodestructiva o la descompensación psicótica, así como para decidir sobre la asignación del tratamiento y el lugar adecuado para los internos. También puede desempeñar un papel importante en la toma de decisiones sobre la libertad condicional o las condiciones específicas para control y supervisión (Echeburúa, Fernández-Montalvo y Corral, 2008; Echeburúa, Sarasua, Zubizarreta, Amor y Corral, 2010).

Las entrevistas clínicas y la información colateral son una vía adecuada para valorar la personalidad en el contexto forense, en donde es muy habitual la simulación. Por ello, sólo es procedente aplicar pruebas psicológicas cuando hay constancia de que un problema psicolegal pueda ser valorado por un test determinado y de que éste tiene las propiedades psicométricas adecuadas, si bien en la mayoría de los casos éstas se han puesto a prueba en poblaciones clínicas (no forenses) (Heilbrun, 1992). En caso contrario, la administración indiscriminada de instrumentos puede suponer malgastar el tiempo o, lo que es peor, obtener unas conclusiones de dudoso rigor con consecuencias jurídicas más o menos graves para el interesado. Las responsabilidades éticas comienzan con una adecuada preparación y continúan con la selección, administración, puntuación e interpretación de los resultados de las pruebas (Esbec y Robles, 2013).

En el DSM-5 figura descrito el trastorno antisocial de la personalidad, que está centrado fundamentalmente en las conductas antisociales (Esbec y Echeburúa, 2010). La psicopatía va más allá de este constructo y está integrada por indicadores conductuales (estilo de vida y faceta antisocial), pero también por rasgos de personalidad (aspectos interpersonales y emocionales). Si bien la psicopatía no figura como tal en el DSM-5, probablemente por una cuestión de política criminal (perder peso como eximente $o$ atenuante al no ser calificada de trastorno mental), en las especificaciones del trastorno antisocial existe la posibilidad de añadir si existen o no manifestaciones psicopáticas.

El instrumento más utilizado en contextos forenses es la Escala de Valoración de la Psicopatía (PCL-R) (Hare, 2003; adaptación española de Torrubia, Poy, Moltó, Grayston y Corral, 2012), especialmente para valorar el riesgo de 
Tabla 3. Organización de los rasgos psicopáticos según la PCL-R (Hare, 2003)

Factor 1

Faceta interpersonal

Factor 1

Faceta emocional

Factor 2

Faceta estilo de vida

Factor 2

Faceta antisocial

Ítems adicionales
1.-Locuacidad/encanto superficial

2.-Egocentrismo/Sensación grandiosa de autovalía

4.-Mentira patológica

5.-Dirección/manipulación

6.-Falta de remordimientos y de culpabilidad

7.-Escasa profundidad de los afectos

8.-Insensibilidad/falta de empatía

16.-Incapacidad para aceptar la responsabilidad de las propias acciones

3.-Necesidad de estimulación/tendencia al aburrimiento

9.-Estilo de vida parasitario

13.-Falta de metas realistas a largo plazo

14.-Impulsividad

15.-Irresponsabilidad

10.-Falta de control conductual

12.-Problemas de conducta precoces

18.-Delincuencia juvenil

19.-Revocación de la libertad condicional

20.-Versatilidad criminal

11.- Conducta sexual promiscua

17.- Varias relaciones de pareja breves conducta criminal. La PCL-R organiza las veinte características definitorias del trastorno en dos factores (rasgos de personalidad e indicadores conductuales). Los primeros se agrupan en la faceta interpersonal y en la faceta emocional; y los segundos, en la faceta del estilo de vida y en la faceta antisocial. Por último, hay dos criterios adicionales que no saturan en ningún factor (Echeburúa y Fernández-Montalvo, 2007; Fernández-Montalvo y Echeburúa, 2008) (tabla 3).

Utilizando criterios específicos de ponderación, cada ítem se puntúa en una escala ordinal de tres puntos (0-12), dependiendo de la presencia e intensidad del mismo en el sujeto a evaluar. El rango es de 0 a 40. Una puntuación superior a 30 sugiere la presencia de una psicopatía y una puntuación superior a 20 sugiere la presencia de tendencias psicopáticas.

El procedimiento de evaluación en sí consiste en una serie de pruebas, en la entrevista estructurada de 125 preguntas y en la investigación sobre el pasado del paciente, incluyendo antecedentes penales, historial médico, educativo y de trabajo, movimiento físico entre lugares y domicilios, historia marital y otras relaciones y entrevistas con tantas personas allegadas al sujeto (familiares, compañeros de trabajo, profesores, amigos, terapeutas, etcétera) como sea posible.

Por último, las personas con trastorno antisocial, psicopatía o con el dominio de antagonismo acusado son propensas a la simulación, siempre que exista un incentivo externo, lo cual suele ocurrir en contextos forenses. Aunque la clave de la evaluación es la exploración clínica y los signos de sospecha (Esbec, 2012), algunas pruebas psicodiagnósticas, como el MMPI-2-RF o el PAI, pueden ser muy útiles para detectarlos.

\section{Conclusiones}

Desde una perspectiva clínica es fundamental evaluar los trastornos de personalidad en el paciente porque tienen una influencia en el manejo y pronóstico terapéutico del caso clínico. Los cuestionarios y las entrevistas estructuradas son los sistemas de medida más utilizados. El grado de concordancia entre los autoinformes y las entrevistas clínicas es todavía bajo, lo que indica una baja fiabilidad en el diagnóstico de estos trastornos.

Si bien hay un cierto acuerdo en la superioridad de las entrevistas (el IPDE o la SCID-II) sobre los cuestionarios (el PID-5, el PAI, el MCMI-III o el MMPI-2) en este campo, las entrevistas requieren un tiempo de administración prolongado y un personal especializado. Por ello, los cuestionarios son los instrumentos a los que más se recurre en la clínica y en la investigación (Esbec y Robles, 2013).

Sin embargo, los autoinformes utilizados en la evaluación de los TP son muy vulnerables a la manipulación debido 
al carácter egosintónico de muchos de ellos. Así, la falta de reconocimiento del problema, la escasa motivación, la simulación o la deseabilidad social son factores que se acentúan en la evaluación de los TP (Echeburúa, FernándezMontalvo y Amor, 2006).

Por ello, las entrevistas clínicas son de mayor utilidad para la evaluación de los TP, sobre todo cuando se cuenta con información colateral (historial del sujeto) y se puede contrastar la información obtenida con otras personas (familiares, por ejemplo). No obstante, las entrevistas también están sujetas a fuentes de distorsión, al carecerse de baremos estandarizados o al depender de las variaciones entre los entrevistadores. Al margen de ello, el diagnóstico psicopatológico requiere siempre de un juicio clínico por parte de un terapeuta, y ello sólo es posible mediante la utilización de entrevistas (Fernández-Montalvo y Echeburúa, 2008).

Las investigaciones que usan cuestionarios tienden a encontrar tasas significativamente más altas de TP que las que recurren a entrevistas estructuradas. Se puede, por ello, concluir que los autoinformes presentan una tendencia a sobrediagnosticar trastornos de personalidad. Las entrevistas clínicas, en cambio, son más estrictas y conservadoras, por lo que las tasas de prevalencia son más bajas.

Las ventajas aparentes de los cuestionarios autoadministrados en el área clínica (su mayor comodidad, el ahorro de costes y de tiempo, etcétera) comportan un detrimento en la calidad de la evaluación y los hace, por tanto, menos fiables en este ámbito.

En resumen, las fuentes de información actualmente disponibles son: a) las entrevistas y el juicio del clínico; b) los cuestionarios autoadministrados; c) los datos colaterales (expedientes e informes); y d) las informaciones complementarias de los familiares o personas que conviven con el paciente. Si bien la validez de cada una de estas fuentes está aún por establecer la utilización conjunta de todas ellas, así como la observación a lo largo del tiempo, parecen potenciar la validez del diagnóstico.

\section{Referencias}

American Psychiatric Association (2013). Diagnostic and Statistical Manual of Mental Disorders ( $5^{\text {th }}$ ed.). Washington, DC: Author.

Anderson, J.L., Sellbom, M., Bagby, R.M., Quilty, L.C., y Veltri, C.O. (2013). On the convergence between PSY-5 domains and PID-5 domains and facets: Implications for assessment of DSM-5 personality traits. Assessment, 20, 286-294.

Ashton, M.C., Lee, K., De Vries, R.E., Hendrickse, J., y Born, M.P. (2012). The maladaptive personality traits of the personality inventory for DSM-5 (PID-5) in relation to the HEXACO personality factors and schizotypy/dissociation. Journal of Personality Disorders, 26, 641-659.
Bastiaansen, L., De Fruyt, F., Rossi, G., Schotte, C., y Hofmans, J. (2013). Personality disorder dysfunction versus traits: structural and conceptual issues. Journal of Personality Disorders, 27, 293-303.

Ben-Porath, Y.S., y Tellegen, A. (2008). MMPI-2-RF (Minnesota Multiphasic Personality Inventory-2 Restructured Form): Manual for administration, scoring, and interpretation. Minneapolis, MN: University of Minnesota Press.

Cardenal, V., y Sánchez, M. P. (2007). Adaptación y baremación al español del Inventario Clínico Multiaxial de Millon-III (MCMI-III). Madrid: Ediciones TEA.

Cloninger, C.R. (2000). A practical way to diagnosis personality disorders. Journal of Personality Disorders, 14, 99-108.

Davis, M.H. (1983). Measuring Individual Differences in Empathy: Evidence for a Multidimensional Approach. Journal of Personality and Social Psychology, 44, 113-126.

Echeburúa, E., y Fernández-Montalvo, J. (2007). Male batterers with and without psychopathy: An exploratory study in Spanish prisons. International Journal of Offender Therapy and Comparative Criminology, 51, 254-263.

Echeburúa, E., Fernández-Montalvo, J., y Amor, P. J. (2006). Psychological treatment of men convicted of gender violence: A pilot-study in Spanish prisons. International Journal of Offender Therapy and Comparative Criminology, 50, 57-70.

Echeburúa, E., Fernández-Montalvo, J., y Corral, P. (2008). ¿Hay diferencias entre la violencia grave y la violencia menos grave contra la pareja?: un análisis comparativo. International Journal of Clinical and Health Psychology, 8, 355-382.

Echeburúa, E., Sarasua, B., Zubizarreta, I., Amor, P. J., y Corral, P. (2010). Variables predictoras del rechazo, abandono y fracaso terapéutico en hombres violentos contra su pareja tratados psicológicamente en un marco comunitario. International Journal of Clinical and Health Psychology, 10, 403-420.

Echeburúa, E., Salaberría, K., y Cruz-Sáez, M. (2014). Aportaciones y limitaciones del DSM-5 desde la Psicología Clínica. Terapia Psicológica, 32, 65-74.

Esbec E. (2012). Trastornos psicóticos. En H. González-Ordí, P. Santamaría y P. Capilla (Eds.), Estrategias de detección de la simulación. Un manual clínico multidisciplinar (pp. 637-675). Madrid: Ediciones TEA.

Esbec, E., y Echeburúa, E. (2010). Violencia y trastornos de la personalidad: implicaciones clínicas y forenses. Actas Españolas de Psiquiatría, $38,249-261$.

Esbec, E., y Echeburúa, E. (2011). La reformulación de los trastornos de la personalidad en el DSM-V. Actas Españolas de Psiquiatría, 39, 1-11.

Esbec, E., y Robles J. L. (2013). Evaluación de los trastornos de la personalidad en Psiquiatría Forense. En S. Delgado y J. M. Maza (Eds.), Psiquiatría Legal y Forense (pp. 141-178). Barcelona: Bosch.

Fernández-Montalvo, J., y Echeburúa, E. (2008). Trastornos de personalidad y psicopatía en hombres condenados por violencia grave contra la pareja. Psicothema, 20, 193-198.

Gore, W. L., y Widiger, T. A. (2013). The DSM-5 dimensional trait model and five-factor models of general personality. Journal of Abnormal Psychology, 122, 816-821.

Gutiérrez-Zotes, J.A., Bayón, C., Montserrat, C., Valero, J., Labad, A., y Cloninger C. R. (2004). Inventario del Temperamento y el CarácterRevisado (TCI-R). Baremación y datos normativos en una muestra de población general. Actas Españolas de Psiquiatría, 32, 8-15.

Hare, R.D. (2003). The Hare Psychopathy Checklist-Revised. Toronto, CA: Multi-Health Systems.

Heilbrun, K. (1992). The role of psychological testing in forensic assessment. Law and Human Behavior, 16, 257-272.

Hopwood, C.J., Wright, A.G., Krueger, R.F., Shade, N., Markon, K.E., y Morey, L. C. (2013). DSM-5 Pathological personality traits and the Personality Assessment Inventory. Assessment, 20, 269-285.

Krueger, R.F., Derringer, J., Markon, K.E., Watson, D., y Skodol, A.E. (2010). Initial construction of a maladaptive personality trait model and inventory for DSM-5. Psychological. Medicine, 42, 1879-1890.

Loinaz, I., Echeburúa, E., y Ullate, M. (2012). Estilo de apego, empatía y autoestima en agresores de pareja. Terapia Psicológica, 30, 61-70. 
López-Ibor, J.J., Pérez Urdániz, A., y Rubio, V. (1996). Examen internacional de los trastornos de personalidad. Madrid: Méditor.

López-Pérez, B., Fernández-Pinto, I. y Abad, F. J. (2008). Test de empatía cognitiva y afectiva. Madrid: Ediciones TEA.

Loranger, A. W. (1995). Personality Disorder Examination (IPDE) Manual. Yonkers, NY: DV Communications.

Melton, G.B., Petrila, J., Poythress, N. G., y Slobogin, C. (2007). Psychological evaluations for the courts: A handbook for mental health professionals and lawyers (3rd ed.). New York: The Guilford Press.

Mestre, V., Frías, M.D., y Samper, P. (2004). La medida de la empatía: análisis del Interpersonal Reactivity Index. Psicothema, 16, 255-260.

Millon, T., Millon, C., Davis, R., y Grossman, S. (2006). MCMI-III Manual. Minneapolis: NCS Pearson.

Morey, L. C. (1991). Personality Assessment Inventory. Professional Manual. Florida, USA: Psychological Assessment Resources, Inc.

Morey, L. C. (2007). Essentials of PAI Assessment. New York: John Wiley and Sons, Inc.

Ortiz-Tallo, M., Santamaría, P., Cardenal, V., y Sánchez, M. P. (2011). Adaptación española del Inventario de Evaluación de la Personalidad (PAI). Madrid: Ediciones TEA.

Rogers, R., Salekin, R. T., y Sewell, K. W. (1999). Validation of the Millon Clinical Multiaxial Inventory for Axis II disorders: Does it meet the Daubert standard? Law and Human Behavior, 23, 425-443.
Santamaría, P. (2009). Evaluación del test MMPI-2-RF. Madrid: Ediciones TEA.

Sellbom, M., Anderson, J.L., y Bagby, R.M. (2013). Assessing DSM-5 Section III personality traits and disorders with the MMPI-2-RF. Assessment, 20, 709-722.

Skodol A.E, Bender, D.S., Oldham, J.M., Clark, L.A., Morey, L.C., Verheul, R., Krueger, R.F., y Siever L.J. (2011). Proposed changes in personality and personality disorder assessment and diagnosis for DSM-5 Part II: clinical application. Journal of Personality Disorders, 7, 23-40.

Spitzer, R.L., Williams, J.B., y Gibbon, M. (1989). Structured Clinical Interview for DSM-III-R, Axis II. SCID-II. Rev. New York, NY: Biometrics Research Department. New York State Psychiatric Institute.

Thomas, K.M., Yalch, M.M., Krueger, R.F., Wright, A.G., Markon, K.E., y Hopwood, C.J. (2013). The convergent structure of DSM-5 personality trait facets and five-factor model trait domains. Assessment, 20, 308-311.

Torrubia, R., Poy, R., Moltó, J., Grayston, P.R., y Corral, S. (2012). PCLR. Escala de Evaluación de Psicopatía de Hare Revisada. Madrid: Ediciones TEA.

Wright, A.G., Pincus, A.L., Hopwood, C.J., Thomas, K.M., Markon, K.E., y Krueger, R.F. (2012). An interpersonal analysis of pathological personality traits in DSM-5. Assessment, 19, 263-275. 